PROCEEDINGS OF THE

AMERICAN MATHEMATICAL SOCIETY

Volume 138, Number 6, June 2010, Pages 2071-2077

S 0002-9939(09)10222-8

Article electronically published on December 9, 2009

\title{
A RESULT ON VALUE DISTRIBUTION OF L-FUNCTIONS
}

\author{
BAO QIN LI
}

(Communicated by Mei-Chi Shaw)

\begin{abstract}
We will establish a theorem on value distribution of L-functions in the Selberg class, which shows how an L-function and a meromorphic function are uniquely determined by their $c$-values and which, as a consequence, proves a result on the unicity of the Riemann zeta function.
\end{abstract}

L-functions, with the Riemann zeta function as a prototype, are important objects in number theory, and value distribution of L-functions has been studied extensively (cf. the recent monograph $[\mathrm{St}$ ] and various references therein). Value distribution of L-functions concerns distribution of the zeros of L-functions $\mathcal{L}$ and, more generally, the $c$-values of $\mathcal{L}$, i.e., the roots of the equation $\mathcal{L}(s)=c$, or the values in the preimage $\mathcal{L}^{-1}(c)=\{s \in \mathbf{C}: \mathcal{L}(s)=c\}$, where and throughout the paper, $s$ denotes the complex variable in the complex plane $\mathbf{C}$ and $c$ denotes a complex value. L-functions can be analytically continued as meromorphic functions in C. It is well-known that a nonconstant meromorphic function in $\mathbf{C}$ is completely determined by five such preimages (see e.g. $[\underline{\mathrm{H}}]$ ), which is a famous theorem due to Nevanlinna and often referred to as Nevanlinna's uniqueness or unicity theorem. Two meromorphic functions $f$ and $g$ in the complex plane are said to share a value $c \in \mathbf{C} \cup\{\infty\}$ IM (ignoring multiplicities) if $f^{-1}(c)=g^{-1}(c)$ as two sets in C. Moreover, $f$ and $g$ are said to share a value $c$ CM (counting multiplicities) if they share the value $c$ and if the roots of the equations $f(s)=c$ and $g(s)=c$ have the same multiplicities. In terms of sharing values, two nonconstant meromorphic functions in $\mathbf{C}$ must be identically equal if they share five values IM, and one must be a Möbius transform of the other if they share four values CM; the numbers "five" and "four" are the best possible, as shown by Nevanlinna (see e.g. $[\mathrm{H}]$ or $[\mathrm{St}]$ ).

This paper concerns the question of how an L-function is uniquely determined in terms of the preimages of complex values, or sharing values. We refer the reader to the monograph $\mathrm{St}$ ] for a detailed discussion on the topic and related works. Throughout the paper, an L-function always means an L-function $\mathcal{L}$ in the Selberg class, which includes the Riemann zeta function $\zeta(s)=\sum_{n=1}^{\infty} n^{-s}$ and essentially those Dirichlet series where one might expect a Riemann hypothesis. Such an Lfunction is defined to be a Dirichlet series $\mathcal{L}(s)=\sum_{n=1}^{\infty} a(n) n^{-s}$, satisfying the following axioms ( $\mathrm{St}]$ and $[\mathrm{Se}]$ ):

(i) Ramanujan hypothesis. $a(n) \ll n^{\varepsilon}$ for every $\varepsilon>0$.

(ii) Analytic continuation. There is a non-negative integer $k$ such that $(s-1)^{k} \mathcal{L}(s)$ is an entire function of finite order.

Received by the editors June 13, 2009, and, in revised form, September 20, 2009.

2010 Mathematics Subject Classification. Primary 30D30, 30D35, 11M06, 11M36.

(C) 2009 American Mathematical Society Reverts to public domain 28 years from publication 
(iii) Functional equation. $\mathcal{L}$ satisfies a functional equation of type

$$
\Lambda_{\mathcal{L}}(s)=\omega \overline{\Lambda_{\mathcal{L}}(1-\bar{s})},
$$

where

$$
\Lambda_{\mathcal{L}}(s)=\mathcal{L}(s) Q^{s} \prod_{j=1}^{K} \Gamma\left(\lambda_{j} s+\nu_{j}\right)
$$

with positive real numbers $Q, \lambda_{j}$ and complex numbers $\nu_{j}, \omega$ with $\operatorname{Re} \nu_{j} \geq 0$ and $|\omega|=1$.

(iv) Euler product hypothesis. $\mathcal{L}(s)=\prod_{p} \exp \left(\sum_{k=1}^{\infty} \frac{b\left(p^{k}\right)}{p^{k s}}\right)$ with suitable coefficients $b\left(p^{k}\right)$ satisfying $b\left(p^{k}\right) \ll p^{k \theta}$ for some $\theta<\frac{1}{2}$, where the product is taken over all prime numbers $p$.

First of all, the number of sharing values can be substantially reduced for the uniqueness of two L-functions, as seen from the following result due to Steuding, which actually holds without the Euler product hypothesis ([St, 152]):

Theorem. If two L-functions $\mathcal{L}_{1}$ and $\mathcal{L}_{2}$ with a $(1)=1$ share a complex value $c \neq \infty C M$, then $\mathcal{L}_{1} \equiv \mathcal{L}_{2}$.

In particular, two L-functions with $a(1)=1$ must be identically equal if they have the same zeros with counting multiplicities. And two L-functions with "enough" common zeros (without counting multiplicities) are expected to be dependent in a certain sense (cf. $[\mathrm{BP}]$ ). Since L-functions are analytically continued as meromorphic functions in the plane, in order to study how an L-function is uniquely determined by preimages of complex values, one should examine the situation involving an arbitrary L-function and an arbitrary meromorphic function. The first observation on this uniqueness problem is that the above theorem no longer holds for an L-function and a meromorphic function. For instance, the function $\zeta$ and $\zeta e^{g}$, where $g$ is any entire function, share $0 \mathrm{CM}$, but they are not identically equal. It is natural to consider two sharing values, i.e., if two sharing values with counting multiplicities would force an L-function and a meromorphic function to be identically equal. This turns out not to be the case either. For instance, consider the function $f=\frac{2 \zeta}{\zeta+1}$. It is then clear that $\zeta$ and $f$ share $0,1 \mathrm{CM}$, but they are not identically equal. Observe, however, when considering L-functions, these functions have only one possible pole at $s=1$, which is implicit in the conditions of the above theorem. Thus, this leads us to consider the natural objects-those meromorphic functions with finitely many poles. The uniqueness theorem (Theorem 1 below) is then established.

Theorem 1. Let $a, b \in \mathbf{C}$ be two distinct values and let $f$ be a meromorphic function in $\mathbf{C}$ with finitely many poles. If $f$ and a nonconstant $L$-function $\mathcal{L}$ share a $C M$ and $b$ IM, then $\mathcal{L} \equiv f$.

The number "two" in Theorem 1 is the best possible, as shown by the above example with $\mathcal{L}=\zeta$ and $f=\zeta e^{g}$.

Corollary 2. Let $a, b, c \in \mathbf{C} \cup \infty$ be three distinct values with $a \in \mathbf{C}$ and $b=\infty$ or $c=\infty$. If $f$ is a meromorphic function in $\mathbf{C}$ that shares a $C M$ and $b, c$ IM with a nonconstant $L$-function $\mathcal{L}$, then $f \equiv \mathcal{L}$.

The number "three" in Corollary 2 is the best possible, as shown by the above example with $\mathcal{L}=\zeta$ and $f=\frac{2 \zeta}{\zeta+1}$. 
In a communication to the author, C.C. Yang asked the following question on the Riemann zeta function (see also $[\mathrm{LY}]$ ):

Question. If $f$ is a meromorphic function in $\mathbf{C}$ that shares two distinct values $a, b$ $\mathrm{CM}$ and $c \neq a, b, 0, \infty \mathrm{IM}$ with the Riemann zeta function $\zeta$, is $f$ equal to $\zeta$ ?

Taking $\mathcal{L}=\zeta$, Corollary 2 shows that $f \equiv \zeta$ if any one of $a, b, c$ is $\infty$ in the above question.

To prove our results, we will employ Nevanlinna theory. For the convenience of the reader who is not familiar with Nevanlinna theory, we list here the notation and results from Nevanlinna theory which will be used in the proofs (see e.g. $[\underline{\mathrm{H}}$ ). Let $f$ be a meromorphic function in $\mathbf{C}$. Then the Nevanlinna characteristic $T(r, f)$ is defined as

$$
T(r, f)=m(r, f)+N(r, f)
$$

where

$$
m(r, f)=\frac{1}{2 \pi} \int_{0}^{2 \pi} \log ^{+}\left|f\left(r e^{i \theta}\right)\right| d \theta, \quad \log ^{+}|x|=\max (0, \log |x|),
$$

and

$$
N(r, f)=\int_{0}^{r} \frac{n(t, f)-n(0, f)}{t} d t+n(0, f) \log r
$$

where $n(t, f)$ denotes the number of poles of $f$ (counting multiplicity) in $|s|<t$. $\bar{N}(r, f)$ is defined in the same way with $n(t, f)$ being replaced by the number of poles of $f$ (ignoring multiplicities) in $|s|<t$. Recall the following known results:

(i) The arithmetic properties of $T(r, f)$ and $m(r, f)$ :

$$
\begin{gathered}
T(r, f g) \leq T(r, f)+T(r, g), \\
T(r, f+g) \leq T(r, f)+T(r, g)+O(1) .
\end{gathered}
$$

The same inequalities hold for $m(r, f)$.

(ii) $T(r, f)$ is an increasing function of $r . f$ is rational if and only if $T(r, f)=$ $O(\log r)$.

(iii) The Nevanlinna first fundamental theorem:

$$
T(r, f)=T\left(r, \frac{1}{f}\right)+O(1) .
$$

(iv) The logarithmic derivative lemma: $m\left(r, \frac{f^{\prime}}{f}\right)=O(\log r)$ if the order

$$
\rho(f):=\limsup _{r \rightarrow \infty} \frac{\log T(r, f)}{\log r}
$$

of $f$ is finite.

(v) The Nevanlinna second fundamental theorem:

$$
(q-2) T(r, f) \leq \sum_{j=1}^{q} \bar{N}\left(r, \frac{1}{f-a_{j}}\right)+S(r, f),
$$

where $a_{j}$ 's are distinct complex values in $\mathbf{C} \cup\{\infty\}$ and $S(r, f)$ denotes a quantity satisfying $S(r, f)=o\{T(r, f)\}$ for all $r$ outside possibly a set of finite Lebesgue measure. If $f$ is of finite order, then $S(r, f)=O(\log r)$. 
Proof of Theorem 1. We first introduce the auxiliary function

$$
F=\frac{Q(\mathcal{L}-a)}{f-a},
$$

where $Q$ is a rational function such that $F$ has neither a pole nor a zero in C. Such a $Q$ does exist since $f$ has only finitely many poles and $\mathcal{L}$ has only one possible pole at $s=1$, and a possible zero or pole of $\frac{\mathcal{L}-a}{f-a}$ may only come from a pole of $\mathcal{L}$ or $f$, in view of the assumption that $\mathcal{L}$ and $f$ share $a \mathrm{CM}$. Thus, $F$ is an entire function without any zeros. Hence, there is an entire function $g$ such that

$$
F=\frac{Q(\mathcal{L}-a)}{f-a}=e^{g} .
$$

We claim that $g$ is linear. To this end, we now show that both $\mathcal{L}$ and $f$ are of order at most one. Suppose that $d$ is the degree of $\mathcal{L}$, i.e., $d=2 \sum_{j=1}^{K} \lambda_{j}$, where $K$ and $\lambda_{j}$ are the numbers in the axiom (iii) (functional equation) of the definition of L-function. Then we have that

$$
T(r, \mathcal{L})=\frac{d}{\pi} r \log r+O(r)
$$

(see [St, p. 150]). It is then easy to see that $\mathcal{L}$ is of order at most 1 . To show that the order $\rho(f)$ of $f$ is at most one, we use the Nevanlinna second fundamental theorem to deduce that

$$
\begin{aligned}
& T(r, f)<\bar{N}\left(r, \frac{1}{f-a}\right)+\bar{N}\left(r, \frac{1}{f-b}\right)+\bar{N}(r, f)+S(r, f) \\
& =\bar{N}\left(r, \frac{1}{\mathcal{L}-a}\right)+\bar{N}\left(r, \frac{1}{\mathcal{L}-b}\right)+\bar{N}(r, f)+S(r, f) .
\end{aligned}
$$

But $f$ has finitely many poles. Thus, $\bar{N}(r, f)=O(\log r)$. Also, by the first fundamental theorem, we have that

$$
\begin{aligned}
& \bar{N}\left(r, \frac{1}{\mathcal{L}-a}\right) \leq T\left(r, \frac{1}{\mathcal{L}-a}\right) \\
& =T(r, \mathcal{L}-a)+O(1) \leq T(r, \mathcal{L})+O(1) .
\end{aligned}
$$

Similarly,

$$
\bar{N}\left(r, \frac{1}{\mathcal{L}-b}\right) \leq T(r, \mathcal{L})+O(1)
$$

Thus, we obtain that

$$
T(r, f) \leq 2 T(r, \mathcal{L})+O(\log r)+S(r, f)
$$

or

$$
(1-o(1)) T(r, f) \leq 2 T(r, \mathcal{L})+O(\log r)
$$

for all $r$ except possibly a set $E$ of finite Lebesgue measure $|E|$. To obtain a bound on the order of $f$, by the definition of order we need an estimate on $T(r, f)$ for all large $r$ without any exceptional set. If $r \in E$, then there exists an $r_{1} \notin E$ such that $0<r_{1}-r<|E|+1$. Recall that the Nevanlinna characteristic is an increasing 
function. Thus, for all large $r$ (in $E$ or not in $E$ ), we always have that

$$
\begin{aligned}
& T(r, f) \leq T(r+|E|+1, f) \\
& \leq(1+o(1))\{2 T(r+|E|+1, \mathcal{L})+O(\log (r+|E|+1))\} \\
& \leq 4 T(r+|E|+1, \mathcal{L})+O(\log (r+|E|+1)) \\
& \leq \frac{4 d}{\pi}(r+|E|+1) \log (r+|E|+1)+O(\log (r+|E|+1)) \\
& =O(r \log r)
\end{aligned}
$$

in view of (2). Hence, we deduce that

$$
\begin{aligned}
& \rho(f)=\limsup _{r \rightarrow \infty} \frac{\log T(r, f)}{\log r} \\
& \leq \limsup _{r \rightarrow \infty} \frac{\log (O(r \log r))}{\log r} \leq 1 .
\end{aligned}
$$

That is, $f$ is of order at most one. Now that both $\mathcal{L}$ and $f$ are of order at most one, it is easy to deduce, by (1), that $F$ is of order at most one. By the Hadamard factorization theorem (see e.g. [BG, p. 384]) we know that in (1), $g$ is linear, as claimed earlier.

Thus, $g=a_{1} s+b_{1}$ for some complex numbers $a_{1}, b_{1}$. It is then easy to verify that

$$
T(r, F)=T\left(r, e^{a_{1} s+b_{1}}\right)=O(r) .
$$

Also, by (1), we see that a zero of $\mathcal{L}-b$ is a zero of $\frac{F}{Q}-1$, since $\mathcal{L}$ and $f$ share $b$. Thus, we deduce, by (3) and the first fundamental theorem, that

$$
\begin{aligned}
& \bar{N}\left(r, \frac{1}{\mathcal{L}-b}\right) \leq N\left(r, \frac{1}{\frac{F}{Q}-1}\right) \leq T\left(r, \frac{1}{\frac{F}{Q}-1}\right) \\
& =T\left(r, \frac{F}{Q}-1\right)+O(1) \\
& \leq T(r, F)+T(r, Q)+O(1) \\
& =O(r)+O(\log r)+O(1)=O(r),
\end{aligned}
$$

in view of the fact that $T(r, Q)=O(\log r)$, since $Q$ is a rational function.

Next, introduce the following auxiliary function:

$$
G=\left(\frac{\mathcal{L}^{\prime}}{(\mathcal{L}-a)(\mathcal{L}-b)}-\frac{f^{\prime}}{(f-a)(f-b)}\right)(f-\mathcal{L}) .
$$

We claim that $G \equiv 0$. Suppose that this is not the case. We will derive a contradiction below.

Assume that $w$ is a zero of $\mathcal{L}-a$ of order $n$ and thus a zero of $f-a$ also of order $n$, since $\mathcal{L}$ and $f$ share $a \mathrm{CM}$. Then one can check that the principal part of the Laurent expansion of $\frac{\mathcal{L}^{\prime}}{(\mathcal{L}-a)(\mathcal{L}-b)}$ at $s=w$ is $\frac{n}{(a-b)(s-w)}$ and the function $\frac{f^{\prime}}{(f-a)(f-b)}$ has the same principal part. Thus, they are canceled out in the expansion of $\frac{\mathcal{L}^{\prime}}{(\mathcal{L}-a)(\mathcal{L}-b)}-\frac{f^{\prime}}{(f-a)(f-b)}$. However, $\mathcal{L}-f$ has a zero at $w$, since $\mathcal{L}$ and $f$ share $a$. This shows that $w$ is zero of $G$.

Similarly, if $w$ is a zero of $\mathcal{L}-b$ of order $n$ and thus a zero of $f-b$ of order $m$, which is possibly different from $n$ since $\mathcal{L}$ and $f$ share $b \mathrm{IM}$, then the principal part of the Laurent expansion of $\frac{\mathcal{L}^{\prime}}{(\mathcal{L}-a)(\mathcal{L}-b)}$ at $s=w$ is $\frac{n}{(b-a)(s-w)}$, while the one 
for $\frac{f^{\prime}}{(f-a)(f-b)}$ is $\frac{m}{(b-a)(s-w)}$. Although these two principal parts in the expansion of $\frac{\mathcal{L}^{\prime}}{(\mathcal{L}-a)(\mathcal{L}-b)}-\frac{f^{\prime}}{(f-a)(f-b)}$ cannot be canceled out, the term $s-w$ in the denominator of the principal parts will be canceled out by the term $s-w$ in the Taylor expansion of $\mathcal{L}-f$, which has a zero at $w$. This shows that $w$ is not a pole of $G$.

The above shows that the only possible poles of $G$ come from the poles of $f$ and $\mathcal{L}$, which are finitely many. Hence, $G$ has at most finitely many poles, which implies that

$$
N(r, G)=O(\log r) .
$$

On the other hand, we have from (1) that

$$
\frac{f-\mathcal{L}}{\mathcal{L}-a}=\frac{f-a-(\mathcal{L}-a)}{\mathcal{L}-a}=\frac{f-a}{\mathcal{L}-a}-1=\frac{Q}{F}-1,
$$

which implies by (3) that

$$
\begin{aligned}
& m\left(r, \frac{f-\mathcal{L}}{\mathcal{L}-a}\right) \leq T\left(r, \frac{f-\mathcal{L}}{\mathcal{L}-a}\right)=T\left(r, \frac{Q}{F}-1\right) \\
& \leq T(r, Q)+T(r, F)+O(1)=O(r),
\end{aligned}
$$

noting that $T(r, Q)=O(\log r)$. Similarly,

$$
\frac{f-\mathcal{L}}{f-a}=\frac{f-a-(\mathcal{L}-a)}{f-a}=1-\frac{\mathcal{L}-a}{f-a}=1-\frac{F}{Q},
$$

which implies that

$$
m\left(r, \frac{f-\mathcal{L}}{f-a}\right) \leq T(r, Q)+T(r, F)+O(1)=O(r) .
$$

Also, by the logarithmic derivative lemma,

$$
m\left(r, \frac{\mathcal{L}^{\prime}}{\mathcal{L}-b}\right)=O(\log r), \quad m\left(r, \frac{f^{\prime}}{f-b}\right)=O(\log r) .
$$

It then follows from (7), (8), (9) and (5) that

$$
\begin{aligned}
& m(r, G)=m\left(r, \frac{f-\mathcal{L}}{\mathcal{L}-a} \frac{\mathcal{L}^{\prime}}{\mathcal{L}-b}-\frac{f-\mathcal{L}}{f-a} \frac{f^{\prime}}{f-b}\right) \\
& \leq m\left(r, \frac{f-\mathcal{L}}{\mathcal{L}-a}\right)+m\left(r, \frac{\mathcal{L}^{\prime}}{\mathcal{L}-b}\right)+m\left(r, \frac{f-\mathcal{L}}{f-a}\right)+m\left(r, \frac{f^{\prime}}{f-b}\right)+O(1) \\
& =O(r),
\end{aligned}
$$

which together with (6) yields that $T(r, G)=O(r)$.

Recall that a zero of $\mathcal{L}-a$ is a zero of $G$ (see the paragraph right after (5)). Thus, we deduce, using the first fundamental theorem, that

$$
\bar{N}\left(r, \frac{1}{\mathcal{L}-a}\right) \leq N\left(r, \frac{1}{G}\right) \leq T\left(r, \frac{1}{G}\right)=T(r, G)+O(1)=O(r) .
$$

We then use the second fundamental theorem again to deduce, in view of (4), that

$$
T(r, \mathcal{L})<\bar{N}\left(r, \frac{1}{\mathcal{L}-a}\right)+\bar{N}\left(r, \frac{1}{\mathcal{L}-b}\right)+\bar{N}(r, \mathcal{L})+O(\log r)=O(r) .
$$

This contradicts (2), unless the degree $d$ in (2) is zero. However, it is known (see [St, p. 113]) that when $d=0, \mathcal{L} \equiv 1$, a contradiction to the assumption that $\mathcal{L}$ is nonconstant. This contradiction shows that $G \equiv 0$, as claimed above. 
We are now ready to get back to our original task of showing that $\mathcal{L} \equiv f$. Suppose that $\mathcal{L} \not \equiv f$. We will arrive at a contradiction below. In fact, by the definition of $G$ in (5), we have the

$$
\frac{\mathcal{L}^{\prime}}{(\mathcal{L}-a)(\mathcal{L}-b)}-\frac{f^{\prime}}{(f-a)(f-b)} \equiv 0 .
$$

Suppose that $w$ is a zero of $\mathcal{L}-b$ of order $n$ and thus a zero of $f-b$ of some order $m$. From the argument in the second paragraph right after (5), we have already seen that the principal part of the Laurent expansion of $\frac{\mathcal{L}^{\prime}}{(\mathcal{L}-a)(\mathcal{L}-b)}$ at $s=w$ is $\frac{n}{(b-a)(s-w)}$, while the one for the function $\frac{f^{\prime}}{(f-a)(f-b)}$ is $\frac{m}{(b-a)(s-w)}$. These two principal parts in the left-hand-side of (10) must be canceled out, which implies that $m=n$. That is, $\mathcal{L}$ and $f$ must share $b$ CM.

Recall from (4) that $\bar{N}\left(r, \frac{1}{\mathcal{L}-b}\right)=O(r)$. The proof of this inequality uses only the fact that $\mathcal{L}$ and $f$ share $a \mathrm{CM}$ and $b$ IM. Now that $\mathcal{L}$ and $f$ share $b \mathrm{CM}$, by symmetry or by exactly the same way, we deduce that $\bar{N}\left(r, \frac{1}{\mathcal{L}-a}\right)=O(r)$. Finally, we use the second fundamental theorem again to deduce that

$$
T(r, \mathcal{L})<\bar{N}\left(r, \frac{1}{\mathcal{L}-a}\right)+\bar{N}\left(r, \frac{1}{\mathcal{L}-b}\right)+\bar{N}(r, \mathcal{L})+O(\log r)=O(r),
$$

which, by (2), implies that $d=0$. But, when $d=0, \mathcal{L}$ must be a constant (see above), a contradiction to the assumption of the theorem. This completes the proof.

Proof of Corollary 2. Suppose that one of $b, c$, say $c$, is $\infty$. Then, $\mathcal{L}$ and $f$ share $c=\infty \mathrm{IM}$, and thus $f$ has only one possible pole, since $\mathcal{L}$ has only one possible pole at $s=1$. Hence $f$, which has only one possible pole, and $\mathcal{L}$ share $a \mathrm{CM}$ and $b$ IM. That is, $f$ and $\mathcal{L}$ satisfy exactly the conditions in Theorem 1 . Therefore, the conclusion of the corollary follows from Theorem 1.

\section{ACKNOWLEDGEMENT}

The author would like to thank the referee for helpful suggestions.

\section{REFERENCES}

[BG] C.A. Berenstein and R. Gay, Complex variables, Springer-Verlag, New York, 1991. MR:1107514 (92f:30001)

[BP] E. Bombieri and A. Perelli, Distinct zeros of L-functions, Acta Arith. 83 (1998), 271-281. MR.1611193 (99a:11102)

[H] W. K. Hayman, Meromorphic functions, The Clarendon Press, Oxford Univ. Press, Oxford, 1964. MR0164038 (29:1337)

[LY] L. Liao and C.-C. Yang, On some new properties of the gamma function and the Riemann zeta function, Math. Nachr. 257 (2003), 59-66. MR1992811 (2004g:33005)

[Se] A. Selberg, Old and new conjectures and results about a class of Dirichlet series, Proceedings of the Amalfi Conference on Analytic Number Theory (Maiori, 1989), E. Bombieri et al. (eds.), Collected papers, Vol. II, Springer-Verlag, 1991, pp. 47-63. MR.1220477 (94f:11085)

[St] J. Steuding, Value-distribution of L-functions, Lecture Notes in Mathematics, 1877, Springer-Verlag, Berlin, 2007. MR2330696 (2008m:11172)

Department of Mathematics, Florida International University, Miami, Florida 33199

E-mail address: libaoqin@fiu.edu 